\title{
Terapia ormonale sostitutiva e osteoporosi postmenopausale
}

\author{
Stefano Lello ${ }^{1}$. Giovanni Scambia ${ }^{1} \cdot$ Anna Capozzi ${ }^{1}$ \\ Accettato: 16 novembre 2021 / Pubblicato online: 21 febbraio 2022 \\ (c) The Author(s) 2022
}

\section{Sommario}

L'osteoporosi postmenopausale è un processo per il quale il tessuto osseo sviluppa una fragilità risultando più suscettibile alle fratture anche per traumi che solitamente non comporterebbero tali eventi. La carenza estrogenica è un punto fondamentale nella patogenesi dell'osteoporosi. La TOS rappresenta un trattamento di scelta nella prevenzione della osteoporosi e delle fratture correlate, soprattutto nei primi anni di postmenopausa.

Parole chiave Osteoporosi postmenopausale $\cdot$ Tibolone $\cdot$ Raloxifene $\cdot$ Badoxifene SERMs $\cdot$ TSEC

\section{Introduzione}

L'osteoporosi postmenopausale consiste in una riduzione della densità minerale ossea (Bone Mineral Density, BMD) e della qualità del tessuto osseo, con un conseguente aumento del rischio di frattura [1]. La carenza estrogenica è primaria nella patogenesi della osteoporosi e della fratture ad essa correlate, per cui appare razionale l'utilizzo della terapia ormonale sostitutiva (TOS) nella prevenzione di questa condizione [2]. La TOS a dosi standard è in grado di prevenire la perdita scheletrica in menopausa, inibendo il riassorbimento osseo mediato dagli osteoclasti e riducendo il tasso del turnover osseo, con riduzione del rischio di frattura $[3,4]$.

\section{Trattamenti ormonali/simil-ormonali sostitutivi e osteoporosi menopausale}

Tra i trattamenti ormonali/simil-ormonali per via sistemica in postmenopausa, esistono: la terapia ormonale sostitutiva (TOS), estrogeni + progestinici nelle donne con utero, soli estrogeni (ET) nelle donne senza utero, il tibolone, i modulatori selettivi del recettore estrogenico ( $\mathrm{Se}$ lective Estrogen Receptor Modulators, SERMs), e il Tissue Selective Estrogen Complex (TSEC: estrogeni coniugati + bazedoxifene).

\footnotetext{
Proposto da S. Migliaccio.

$\bowtie$ S. Lello

lello.stefano@gmail.com

1 Dipartimento Salute Donna e Bambino, Fondazione Policlinico Gemelli-IRCCS, Roma, Italia
}

\section{Terapia ormonale sostitutiva e tessuto osseo}

La TOS ha diversi dosaggi [5]: standard $(0,625 \mathrm{mg}$ di estrogeni coniugati, EC o equivalenti), low-dose $(0,45 \mathrm{mg}$ di EC o equivalenti), ultra low-dose ( $0,3 \mathrm{mg}$ di EC o equivalenti).

Gli estrogeni regolano il metabolismo scheletro, modulando la funzione e la sopravvivenza di osteociti (riduzione apoptosi), osteoclasti (aumento della apoptosi, riduzione della differenziazione indotta dal RANK-ligando) e osteoblasti (riduzione della apoptosi), con riduzione del rimodellamento osseo e riduzione del tasso di riassorbimento. Attraverso tali meccanismi, gli estrogeni proteggono la massa ossea [6]. Durante la TOS gli indici di riassorbimento tendano a ridursi significativamente, con aumento di BMD [7]. Una metanalisi, pubblicata nel 2002 [8], su 57 trial randomizzati e controllati con placebo, riportava BMD lombare, di collo femorale e avambraccio aumentata significativamente dopo 1 e 2 anni di TOS standard. Anche le basse dosi di estrogeni sono attive sull'osso; $0,3 \mathrm{mg}$ di estrogeni coniugati proteggono la BMD lombare e femorale rispetto al placebo dopo 2 anni di trattamento [9]. Nello studio HOPE, le dosi di 0,3, 0,45 e $0,625 \mathrm{mg} /$ die di EC, associate o meno al progestinico medrossiprogesterone acetato (MPA), hanno ridotto significativamente $(p<0,01)$ i livelli del marker di riassorbimento N-telopeptide del collagene di tipo 1 (NTX) dopo 6, 12, 18 e 24 mesi di osservazione di osservazione; anche il marker di neoformazione osteocalcina è stato ridotto in modo significativo $(p<0,01)[10]$.

Quindi, per la BMD, dosi inferiori a quelle standard possono essere efficaci nel prevenire la perdita ossea in donne anziane; comunque, nello studio HOPE [10], il sub-studio 
sull'osso ( $\mathrm{n}=822)$, durato due anni, ha mostrato BMD lombare nei gruppi in trattamento attivo significativamente aumentata versus baseline $(p<0,001)$, con differenza significativa versus placebo $(p<0,001)$. L'aggiunta di $2,5 \mathrm{mg}$ di MPA a $0,45 \mathrm{mg}$ e $0,625 \mathrm{mg}$ di EC ha aumentato significativamente la BMD lombare versus stesse dosi di EC senza progestinico $(p<0,05)$. Quindi, anche basse dosi di EC ed EC+MPA prevengono la perdita ossea lombare. Infine, lo studio HOPE mostra come anche la BMD a livello del femore totale viene protetta da basse dosi di estrogeni. Indipendentemente dalla via di somministrazione, gli estrogeni sono attivi sull'osso; nel 1999, Weiss e collaboratori, in uno studio multicentrico, randomizzato e controllato con placebo, hanno dimostrato come diversi dosaggi di estradiolo transdermico con un range da $25 \mathrm{mcg}$ (low-dose) a $100 \mathrm{mcg} / \mathrm{die}$ (high dose) sono in grado di aumentare(??) la BMD in donne in postmenopausa, a livello lombare femorale, rispetto al placebo [11]. Infatti, dopo 24 mesi di osservazione le dosi di estradiolo di 0,025, 0,05, 0,06 e 1,0 mg/die hanno aumentato la BMD lombare di $2,37 \%, 4,09 \%, 3,28 \%$ e $4,70 \%$, rispettivamente; anche a livello femorale è stato riportato una BMD aumentata di $0,26 \%, 2,85 \%, 3,05 \%$ e $2,03 \%$, rispettivamente. Nello studio Women's Health Initiative (WHI) [12], randomizzato e controllato con placebo, in donne postmenopausali, ha dimostrato come la TOS standard riduce significativamente, versus placebo, il rischio di fratture vertebrali $(\mathrm{HR}=0,66$; IC 95\%: 0,45-0,98) e femorali $(\mathrm{HR}=0,66$; IC 95\%: 0,44-0,98).

\section{Tibolone e tessuto osseo}

Il tibolone è un composto che viene trasformato in tre metaboliti principali, due con attività estrogeniche e uno con attività di tipo progestinica/androgenica. Uno studio prospettico [13], non-randomizzato, su effetto del tibolone sulla perdita ossea, ha coinvolto 110 donne in postmenopausa da non più di 3 anni; 59 donne sono state trattate con tibolone $(2,5$ $\mathrm{mg} / \mathrm{die}$ ) per 10 anni e 51 donne rappresentavano il gruppo di controllo. La densitometria a livello lombare e femorale è stata effettuata annualmente. Dopo 10 anni nel gruppo trattato con tibolone si è evidenziato una BMD aumentata significativamente $(p<0,0001) v s$ i livelli di base a lombari $(+4,8 \%)$ e del collo femorale $(+3,7 \%)$. Nel gruppo controllo la BMD è diminuita significativamente versus i valori basali (-8,5\% rachide lombare; $-8,9 \%$ collo del femore). Nello studio LIFT [14], il tibolone (al dosaggio di $1,25 \mathrm{mg} / \mathrm{die}$ ) in una popolazione di età media di 68 anni, ha ridotto il rischio di frattura vertebrale $(\mathrm{RH}=0,55$; IC 95\%: $0,41-0,74) \mathrm{e}$ non vertebrale $(\mathrm{RH}=0,74$; IC 95\%: 0,58-0,93), in maniera sovrapponibile a estrogeni, bifosfonati e raloxifene.

\section{Modulatori selettivi del recettore estrogenico e tessuto osseo}

I SERMs sono molecole non ormonali che si legano al recettore estrogenico con effetti di tipo estrogenico su alcuni tessuti (osso) e di tipo non estrogenico su altri tessuti (mammella). Il raloxifene (RLX), in uno studio della durata di tre anni [15], randomizzato e controllato con placebo, condotto su 7705 donne in postmenopausa da almeno due anni alla dose di $60 \mathrm{mg} / \mathrm{die}$, ha aumentato la BMD vertebrale e femorale e ridotto significativamente il rischio di frattura vertebrale sia nel gruppo con fratture prevalenti $(\mathrm{RR}=$ 0,70; IC 95\%: 0,56-0,86) sia nel gruppo senza fratture prevalenti (RR = 0,45; IC 95\%: 0,29-0,71; il trattamento con RLX non ha ridotto, rispetto al placebo, il rischio di fratture femorali. Riguardo la prevenzione dell'osteopenia/osteoporosi, RLX riduce significativamente il passaggio dalla condizione di osso normale a osteopenia, a livello vertebrale $(\mathrm{RR}=0,23$; IC 95\%: 0,00-0,81) e femorale, seppur soltanto come trend ( $\mathrm{RR}=0,43$; IC 95\%: 0,11-1,06); anche il passaggio da osteopenia a osteoporosi viene ridotto da RLX $(\mathrm{RR}=0,13$; IC 95\%: 0,00-0,37) per la colonna, mentre nessuna paziente ha sviluppato osteoporosi a livello femorale [16]. Dopo 4 anni di follow-up, il numero dei casi di cancro mammario (end-point secondario dello studio; invasivo e non-invasivo) osservato nel gruppo RLX, presentava una incidenza ridotta del $62 \%$ rispetto al placebo $(R R=0,38$; 95\% CI: 0,24-0,58) [17]. Il bazedoxifene (BZA) è un derivato del RLX per sostituzione del core benzotiofenico con anello indolico, che si lega a $\mathrm{ER} \alpha$ e $\mathrm{ER} \beta$ con alta affinità. $\mathrm{Si}$ comporta come antagonista a livello dell'utero (diminuzione della stimolazione endometriale estrogeno-dipendente) e della mammella (inibizione della proliferazione cellulare). BZA ha dimostrato, in uno studio della durata di 2 anni [18] controllato versus placebo e trattamento attivo (RLX), di ridurre la perdita ossea e il turnover osseo in donne in post-menopausa con BMD normale o basso (T-score a livello vertebrale o femorale tra -1 e $-2,5$ ), in maniera sovrapponibile al RLX e significativamente differente rispetto al placebo. In un altro studio [19], BZA ha ridotto l'incidenza di nuove fratture vertebrali in una popolazione di donne in post-menopausa osteoporotiche; le pazienti avevano assunto quotidianamente BZA $20 \mathrm{mg}$, BZA $40 \mathrm{mg}$ RLX $60 \mathrm{mg}$, o placebo. L'incidenza di nuove fratture vertebrali a 3 anni di trattamento è stata significativamente ridotta con BZA 20 mg (HR = 0,58; IC 95\%: 0,38-0,89; Riduzione dei Rischio Relativo, RRR $=42 \%)$, BZA $40 \mathrm{mg}(\mathrm{HR}=0,63$; IC 95\%: 0,42-0,96; RRR = 37\%), RLX $60 \mathrm{mg}(\mathrm{HR}=0,58$; IC 95\%: $0,38-0,89$; RRR $=42 \%)$; BZA non riduceva le fratture non-vertebrali. In un'analisi post-hoc in soggetti ad alto rischio (T-score collo femorale di $-3,0$ o minore, 1 o più fratture vertebrali o gravi, fratture vertebrali multiple), BZA 
$(20 \mathrm{mg} / \mathrm{die})$ ha ridotto significativamente il rischio di fratture non-vertebrali versus RLX $60 \mathrm{mg}(p=0,05)$ e placebo $(p=0,02)$.

\section{Tissue selective estrogen complex e tessuto osseo}

TSEC è l'associazione tra EC $(0,45 \mathrm{mg} / \mathrm{die})$ e BZA (20 $\mathrm{mg} / \mathrm{die}$ ). In uno studio [20] randomizzato e controllato con placebo e con trattamento attivo (RLX) si è valutato l'effetto del TSEC sul tessuto osseo. La popolazione di studio era costituita da donne entro 1-5 anni di postmenopausa e donne dopo i 5 anni dall'inizio della menopausa. TSEC ha mostrato, indipendentemente dagli anni di post-menopausa, di aumentare la BMD lombare significativamente a 12 e 24 mesi dal baseline versus placebo $(p<0,001)$; così come versus $\operatorname{RLX}(p<0,05)$ nelle donne che erano in postmenopausa da 1-5 anni. TSEC ha determinato un aumento significativo della BMD femorale a 12 e 24 mesi dal baseline rispetto alla diminuzione osservata con il placebo in donne che erano oltre i 5 anni di postmenopausa ( $p<0,01$ ) e in donne entro 1-5 anni dall'inizio della menopausa. TSEC ha ridotto il rimodellamento osseo come testimoniato dai cambiamenti dei markers biochimici dell'osso rispetto al baseline ( $p<0,001$ per osteocalcina e $\mathrm{C}$-telopeptide) versus placebo nelle donne entro 1-5 anni dall'inizio della menopausa.

\section{TOS e osteoporosi: linee guida}

La International Menopause Society (IMS) nel 2016 [21] ha definito la TOS come la terapia più appropriata per la prevenzione delle fratture nel primo periodo della menopausa. La North American Menopause Society (NAMS), nel 2017 ha indicato la TOS come terapia primaria per le prevenzione della perdita ossea e delle fratture in donne in postmenopausa, di età inferiore ai 60 anni o entro 10 anni dall'inizio della menopausa, ad elevato rischio di osteoporosi o di fratture [2].

Un documento di Consenso del 2018, prodotto dalla Società Italiana di Endocrinologia (SIE), dalla Società Italiana dell'Osteoporosi, del Metabolismo Minerale e delle Malattie dello Scheletro (SIOMMMS), e dalla Società Italiana di Ginecologia ed Ostetricia (SIGO) [22], concludeva che la TOS in postmenopausa dovrebbe essere considerata come una delle terapie di prima linea per la prevenzione dell'osteoporosi e delle fratture correlate all'osteoporosi, specialmente nelle donne in postmenopausa di età più giovane di 60 anni o entro i 10 anni dall'inizio della menopausa, grazie al profilo di rischio estremamente basso e a un rapporto beneficio/rischio favorevole.

\section{Conclusioni}

In base a quanto riportato, risulta chiaro come per le basi fisiopatologiche dell'osteoporosi postmenopausale (ruolo della carenza estrogenica), per il meccanismo di azione degli estrogeni e del tibolone (ma anche dei SERMs) somministrati come trattamento (effetto su osteoclasti, osteoblasti e osteociti), e per gli effetti clinici (riduzione della perdita di $\mathrm{BMD}$, riduzione del rischio di frattura), la TOS e il tibolone si pongono tra le terapie di prima scelta nel primo periodo della fase postmenopausale (con inizio della somministrazione in pazienti entro 10 anni dall'inizio della menopausa o prima dei 60 anni di età) per le prevenzione dell'osteoporosi e delle fratture ad essa correlate [5].

Informazioni Supplementari La versione online contiene materiale supplementare disponibile su https://doi.org/10.1007/s40619-02201030-7.

\section{Dichiarazioni etiche}

Conflitto di interesse Gli autori dichiarano di non avere conflitti di interesse.

Consenso informato Lo studio presentato in questo articolo non ha richiesto sperimentazione umana.

Studi sugli animali Gli autori di questo articolo non hanno eseguito studi sugli animali.

Open Access This article is licensed under a Creative Commons Attribution 4.0 International License, which permits use, sharing, adaptation, distribution and reproduction in any medium or format, as long as you give appropriate credit to the original author(s) and the source, provide a link to the Creative Commons licence, and indicate if changes were made. The images or other third party material in this article are included in the article's Creative Commons licence, unless indicated otherwise in a credit line to the material. If material is not included in the article's Creative Commons licence and your intended use is not permitted by statutory regulation or exceeds the permitted use, you will need to obtain permission directly from the copyright holder. To view a copy of this licence, visit http://creativecommons.org/licenses/by/4.0/.

\section{Bibliografia}

1. NIH Consensus Development Panel on Osteoporosis Prevention, Diagnosis, and Therapy (2001) Osteoporosis prevention, diagnosis, and therapy. JAMA 285(6):785-795

2. The NAMS 2017 Hormone Therapy Position Statement Advisory Panel (2017) The 2017 hormone therapy position statement of The North American Menopause Society. Menopause 24(7):728-753

3. Cauley JA, Robbins J, Chen Z (2003) Women's Health Initiative Investigators et al. Effects of estrogen plus progestin on risk of fracture and bone mineral density: the Women's Health Initiative randomized trial. JAMA 290:1729-1738

4. Anderson GL, Limacher M, Assaf AR et al (for the Women's Health Initiative Steering Committee) (2004) Effects of conjugated estrogen in postmenopausal women with hysterectomy: the Women's Health Initiative randomized controlled trial. JAMA 291:1701-1712 
5. Cagnacci A, Gambacciani M, Gallo M, Lello S (for the Executive Committee of The Italian Society of Menopause (SIM) and The Italian Society of Gynecology of Third Age (SIGiTE)) (2019) Recommendations on menopausal hormone replacement therapy. Minerva Ginecol 71(6):395-403

6. Khosla S, Oursler MJ, David G et al (2012) Estrogen and the skeleton. Trends Endocrinol Metab 23(11):576-581

7. Bjarnason NH, Hassager C, Christiansen C (1998) Postmenopausal bone remodelling and hormone replacement. Climacteric 1(1):72-79

8. Wells G, Tugwell P, Shea B et al (2002) Meta-analyses of therapies for postmenopausal osteoporosis. V. Meta-analysis of the efficacy of hormone replacement therapy in treating and preventing osteoporosis in postmenopausal women. Endocr Rev 23:529-539

9. Genant HK, Lucas J, Weiss S et al (1997) Low-dose esterified estrogen therapy: effects on bone, plasma estradiol concentrations, endometrium, and lipid levels. Estratab/Osteoporosis Study Group. Arch Intern Med 157:2609-2615

10. Lindsay R, Gallagher JC, Kleerekoper M et al (2002) Effect of lower doses of conjugated equine estrogens with and without medroxyprogesterone acetate on bone in early postmenopausal women. JAMA 287:2668

11. Weiss SR, Ellman H, Dolker M (for the Transdermal Estradiol Investigator Group) (1999) A randomized controlled trial of four doses of transdermal estradiol for preventing postmenopausal bone loss. Obstet Gynecol 94:330-336

12. Writing Group for the Women's Health Initiative Investigators (2002) Risks and benefits of estrogen plus progestin healthy postmenopausal women. JAMA 288:321-333

13. Rymer J, Robinson J, Fogelman I (2002) Ten years of treatment with tibolone $2,5 \mathrm{mg}$ daily: effects on bone loss in postmenopausal women. Climacteric 5(4):390-398

14. Cummings SR, Ettinger B, Delmas PD et al (for the LIFT Trial Investigators) (2008) The effects of tibolone in older postmenopausal women. N Engl J Med 359:697-708
15. Ettinger B, Black D, Mitlak BH et al (1999) Reduction of vertebral fracture risk in postmenopausal women with osteoporosis treated with raloxifene. JAMA 282:637-645

16. Jolly EE, Bjarnason NH, Neven P et al (2003) Prevention of osteoporosis and uterine effects in postmenopausal women taking raloxifene for 5 years. Menopause 10(4):337-344

17. Cauley JA, Norton L, Lippman ME (2001) Continued breast cancer risk reduction in postmenopausal women treated with raloxifene: 4-year results from the MORE trial. Multiple outcomes of raloxifene evaluation. Breast Cancer Res Treat 65(2):125-134

18. Miller PD, Chines AA, Christiansen CC et al (2008) Effects of bazedoxifene on BMD and bone turnover in postmenopausal women: 2-yr results of a randomized, double-blind, placebo-, and active-controlled study. J Bone Miner Res 23(4):525-535

19. Silverman SL, Christiansen C, Genant HK et al (2008) Efficacy of bazedoxifene in reducing new vertebral fracture risk in postmenopausal women with osteoporosis: results from a 3-year, randomized, placebo-, and active-controlled clinical trial. J Bone Miner Res 23:1923-1934

20. Lindsay R, Gallagher JC, Kagan R et al (2009) Efficacy of tissue-selective estrogen complex (TSEC) of bazedoxifene/conjugated estrogens (BZA/CE) for osteoporosis prevention in at-risk postmenopausal women. Fertil Steril 92:1045-1052

21. Baber RJ, Panay N, Fenton A (for the IMS Writing Group) (2016) 2016 IMS recommendations on women's midlife health and menopause hormone therapy. Climacteric 19:109-150

22. Vignozzi L, Malavolta N, Villa P et al (2019) Consensus statement on the use of HRT in postmenopausal women in the management by SIE, SIOMMMS, and SIGO. J Endocrinol Invest 42:609-618

Nota della casa editrice Springer Nature rimane neutrale in riguardo alle rivendicazioni giurisdizionali nelle mappe pubblicate e nelle affiliazioni istituzionali. 